\title{
Chemical Analysis of B stars within 9-11 kpc from the Galactic Center
}

\author{
Maria Isela Zevallos Herencia and Simone Daflon
}

Observat'orio Nacional,

Rua General José Cristino 77, CEP:20921.400, São Cristóvão, Rio de Janeiro, Brazil

email: mzevallos@on.br; daflon@on.br

\begin{abstract}
Radial gradients of metallicity are supported by observations of different young objects in the Galactic thin disk. The shape of the abundance distributions, however, is not completely constrained. Some works describe the abundance distributions as a function of the Galactocentric distance $\mathrm{R}_{G}$ by linear fits with a single slope. On the other hand some analyses of open clusters, cepheids and OB stars suggest a discontinuity in the abundance distributions around $\mathrm{R}_{G}=10 \mathrm{kpc}$. In this work we analyse a sample of $13 \mathrm{~B}$ stars members of four open clusters located within $\mathrm{R}_{G}=9-11 \mathrm{kpc}$ in order to better constrain the chemical distribution in this region of the disk.
\end{abstract}

Keywords. abundances, early-type stars

\section{Introduction}

Radial gradients of metallicity are important constraints to the chemical evolution models. Observations of different classes of young objects show that the elemental abundances decrease from the Galactic Center towards the edge of the Galactic disk. The radial distribution of the chemical properties of the Galaxy can be studied from B stars and photoionized nebula. Concerning the stellar studies, Daflon \& Cunha (2004) analyzed 69 Galactic OB stars from $\mathrm{R}_{G}=4.7-13 \mathrm{kpc}$, and found a radial metallicity gradient with a slope of $-0.042 \mathrm{dex} / \mathrm{kpc}$. However some objects of the sample at $\mathrm{R}_{G} \sim 10 \mathrm{kpc}$ show abundance values lower than expected for that region of the Galactic Plane. Some results from open clusters (Twarog et al. 1997) and in cepheids (Caputo et al. 2001; Andrievsky et al. 2004) also suggest a discontinuity in the abundance distribution of the Galactic disk in that region. The sample of Daflon \& Cunha (2004) is not enough (especially at $\mathrm{R}_{G} \sim$ $10 \mathrm{kpc}$, where only five stars were analysed) to conclude if such discontinuity really exists or if this minimun in the abundance distribution is an artifact. Concerning the studied elements, nebular analyses are focalized in $\mathrm{O}, \mathrm{S}, \mathrm{Ar}$ and $\mathrm{Ne}$ abundances and rarely in $\mathrm{He}$ and N. In the case of B stars, despite of showing intense He lines in their spectra, most of the works are focused in metals. In both cases the distribution of He abundance, a key element for models of Galactic chemical evolution, is not well established. The objective of this work is to analyze the discontinuity of the abundance gradient at $\mathrm{R}_{G} \sim 9-11 \mathrm{kpc}$, beginning with helium.

\section{The Sample}

Our sample consists of 13 main sequence B stars members of the clusters NGC 2264 $\left(\mathrm{R}_{G}=10.3 \mathrm{kpc}\right)$, NGC $2362\left(\mathrm{R}_{G}=9.3 \mathrm{kpc}\right)$, NGC $2367\left(\mathrm{R}_{G}=9.8 \mathrm{kpc}\right)$ and NGC 2384 $\left(\mathrm{R}_{G}=9.8 \mathrm{kpc}\right)$. The spectra were obtained with spectrograph FEROS + ESO $2.2 \mathrm{~m}$ telescope (under the agreement ESO-ON). 


\section{Analysis}

The effective temperature $\left(\mathrm{T}_{e f f}\right)$ was calculated from a photometric calibration for the reddening free parameter Q (Daflon et al. 1999). The surface gravity ( $\log g$ ) was derived from the fits of theoretical wings of $\mathrm{H} \gamma$ calculated in non-LTE to the observed profiles. We determined non-LTE Helium abundance $(\log \varepsilon(\mathrm{He}))$ for stars with vsini $<150 \mathrm{~km} / \mathrm{s}$, using 9 lines of HeI. The He abundance were determined from spectral synthesis with SYNPLOT, interpolating in the grids of non-LTE model atmospheres OSTAR2002 and BSTAR2006, calculated with the program TLUSTY. We calculated He abundances for three values of microturbulence $(\xi=0,5$ and $10 \mathrm{~km} / \mathrm{s})$ and chose the $\xi$ value that produce an He abundance independent of the line strenght (Fig. 1 (left)). In Fig. 1 (right) are shown the preliminary results of the He abundances as a function of $\mathrm{T}_{\text {eff }}$ for the stars of the sample. From this figure, it seems to exist a trend of the He abundances with $\mathrm{T}_{\text {eff }}$. This result still needs further investigation.
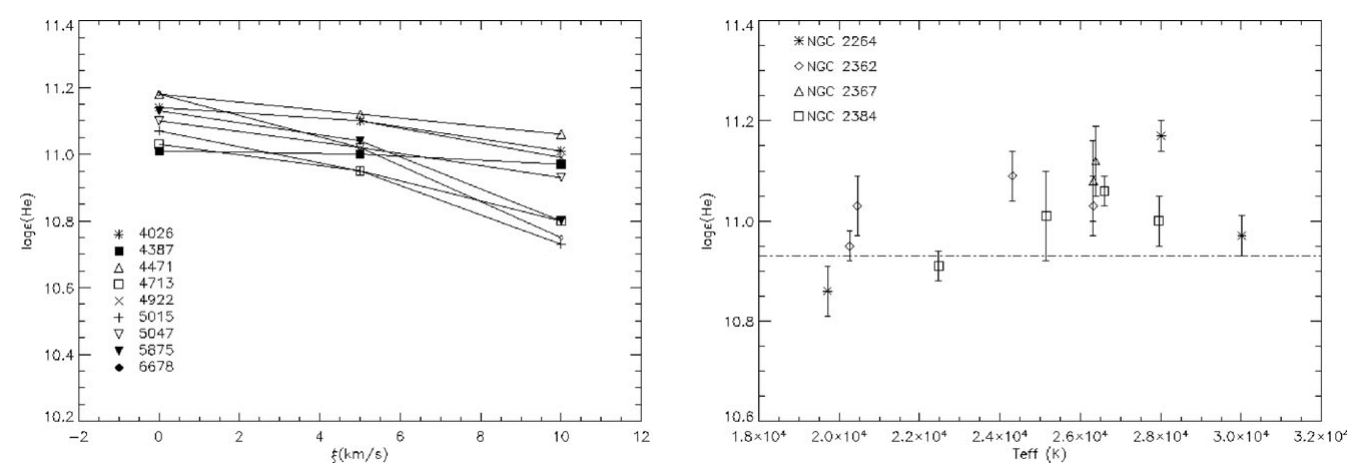

Figure 1. Variation of He abundance with $\xi$ for the star CD-24 5180 (left). $\log \varepsilon(\mathrm{He})$ vs. $\mathrm{T}_{\text {eff }}$ for the sample stars (right) with their respective line-to-line scatter. The dotted line represents the Solar He abundance $(10.93 \pm 0.01)$ from Asplund et al. 2006 .

\section{Conclusions and next steps}

The He abundances obtained are slightly higher than the solar value, except for two stars of the sample, HD47732 and CPD-20 2379. The mean He abundances for each cluster are $11.00 \pm 0.04($ NGC 2264), $11.02 \pm 0.05$ (NGC 2362), $11.10 \pm 0.08$ (NGC 2367 ) and $10.99 \pm 0.05$ (NGC 2384). Our analysis will be extended by the addition of 25 B stars members of the clusters NGC $2439\left(\mathrm{R}_{G}=10.5 \mathrm{kpc}\right)$ and NGC $2467\left(\mathrm{R}_{G}=8.6\right.$ $\mathrm{kpc}$ ); and the sample of Daflon \& Cunha (2004). We will derive non-LTE abundances of $\mathrm{CNO}, \mathrm{Mg}, \mathrm{Si}$ and $\mathrm{S}$ for the sample stars within 9-11 kpc in order to re-compute the radial abundance gradients for these elements.

\section{References}

Andrievsky, S. M., Luck, R. E., Martin, P., \& Lépine, J. R. D. 2004, A\&A, 413, 159

Asplund, M., Grevesse, N., \& Sauval, A. J. 2006, Nuclear Physics A, 777, 1

Caputo, F., Marconi, M., Musella, I., \& Pont, F. 2001, A\&A, 372, 544

Daflon, S., Cunha, K., \& Becker, S. 1999, ApJ, 522, 950

Daflon, S. \& Cunha, K. 2004, ApJ, 617, 1115

Lanz, T. \& Hubeny, I. 2007, ApJS, 169, 83

Twarog Bruce, A., Ashman Keith, M., \& Anthony-Twarog Barbara, J. 1997, AJ, 114, 2556 